Utah State University

DigitalCommons@USU

\title{
Examining the Feasibility and Effectiveness of Online Acceptance and Commitment Therapy Self-Help in a Quasi-Stepped Care Model: A Pilot Study
}

\author{
Clarissa W. Ong \\ Utah State University, clarissa.ong@usu.edu \\ Carina L. Terry \\ Utah State University \\ Michael Levin \\ Utah State University, michael.levin@usu.edu \\ Michael P. Twohig \\ Utah State University, michael.twohig@usu.edu
}

Follow this and additional works at: https://digitalcommons.usu.edu/psych_stures

Part of the Psychology Commons

\section{Recommended Citation}

Ong, C. W., Terry, C. L., Levin, M. E., \& Twohig, M. P. (in press). Examining the feasibility and effectiveness of online acceptance and commitment therapy self-help in a quasi-stepped care model. Psychological Services.

This Article is brought to you for free and open access by the Psychology Student Works at DigitalCommons@USU. It has been accepted for inclusion in Psychology Student Research by an authorized administrator of DigitalCommons@USU. For more information, please contact digitalcommons@usu.edu.

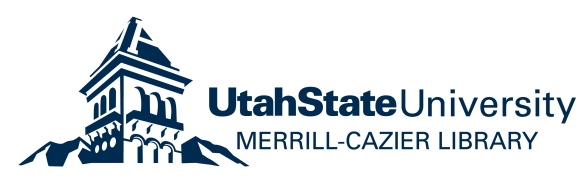


Examining the Feasibility and Effectiveness of

Online Acceptance and Commitment Therapy Self-Help in a Quasi-Stepped Care Model:

\author{
A Pilot Study \\ Clarissa W. Ong \\ Carina L. Terry \\ Michael E. Levin \\ Michael P. Twohig
}

Utah State University

Corresponding author:

Clarissa W. Ong

Department of Psychology

Utah State University

2810 Old Main Hill

Logan, UT 84322-2810

clarissa.ong@usu.edu

Declarations of interest: none.

CRediT author statement: CWO: Conceptualization, writing, formal analysis, investigation. CLT: Writing, investigation. MEL, MPT: Conceptualization, writing, investigation, supervision.

September 15, 2021. This paper has not yet been published. 


\begin{abstract}
The global burden of mental illness and limited resources make increasing the efficiency of available mental healthcare resources especially crucial. One way this can be done is a stepped care approach to treatment. To test the viability of using internet-based self-help in a stepped care model, we examined the feasibility, acceptability, and effectiveness of an online self-help acceptance and commitment (ACT) program prior to starting in-person therapy at a university community clinic $(N=51)$. Online program acceptability was at least moderate. There was clinically significant change in 75.9 to $79.3 \%$ and reliable change in 31.0 to $100 \%$ of our sample on three of four outcomes of interest (e.g., symptom impairment) after the online program. In addition, 60 to $100 \%$ reliably improved from the online program as well as from subsequent treatment on three of four outcomes, indicating that most of our sample progressed through the steps of care effectively and benefited from the quasi-stepped care approach. Scores also indicated positive overall effects of the online program and in-person therapy. Our findings tentatively support the use of low-intensity resources like online self-help programs to reduce therapist burden in outpatient clinics by initiating client progress before intake. Limitation to this approach and the study are discussed.
\end{abstract}

Keywords: stepped care, anxiety, self-help, internet-based, acceptance and commitment therapy 


\section{Impact Statement}

This study examined the utility of offering clients on a clinic waitlist an eight-session online self-help program before starting in-person therapy. Although most participants experienced significant gains in the majority of outcomes assessed, we observed relatively high attrition, suggesting that strategies to increase user engagement with the online program are needed. 
Examining the Feasibility and Effectiveness of

Online Acceptance and Commitment Therapy Self-Help in a Quasi-Stepped Care Model:

\section{A Pilot Study}

The lifetime prevalence of any mental disorder is $47.4 \%$ (Kessler et al., 2007), and that of the most common presentation, major depression, is $16.6 \%$ (Kessler et al., 2012). Meanwhile, the global burden of mental illness is estimated to account for $32.4 \%$ of years lived with disability and $13.0 \%$ of disability-adjusted life-years (Vigo et al., 2016). Thus, psychological conditions are not only common but also account for a sizable portion of global disability. Yet, mental healthcare systems are notoriously under-funded (Vigo et al., 2016) and the resources available to manage treatment of mental health conditions do not match current needs.

To compensate for this gap, mental healthcare systems can try to maximize the efficiency of available resources, so they are able to serve more clients without relying on additional funding. One method to accomplish this is triaging clients using a stepped care model. In a stepped care model, clients receive the least resource-intensive treatment to begin (e.g., psychoeducation groups, self-help books) and are "stepped up" to more resource-demanding interventions if no improvement in observed (Bower \& Gilbody, 2005). That way, more costly and burdensome treatments are reserved for clients who need them most. Some stepped care models have been found to be as effective as (Nordgreen et al., 2016; Seekles et al., 2011) and cheaper than traditional approaches (Ho et al., 2016; Mohr et al., 2019). However, the content of stepped care interventions varies (e.g., guided self-help vs. therapist-led psychoeducation group), so it is possible that other iterations of the stepped care model would not reproduce these effects.

One lower-intensity treatment option entails using self-help interventions wherein people seeking treatment can learn key concepts and skills without seeing a therapist in person. Self- 
help interventions have generally been found to be effective for targeted outcomes (e.g., anxiety in anxiety disorders), even for patients with physical conditions (Matcham et al., 2014; van't Hof et al., 2009). Especially with the proliferation of the internet, one way to potentially increase the accessibility and efficacy of self-help interventions is to integrate technology (e.g., automating treatment components, sharing via the internet). Web-based self-help interventions (guided and unguided) have shown significant positive effects on symptoms, such as suicidality and depression (Buntrock et al., 2016; van Spijker et al., 2012; van Straten et al., 2008). In addition, guided internet-based treatment (i.e., the bulk of care is delivered via the online intervention and the designated provider only provides low-intensity support) appears to be as effective as faceto-face therapy (e.g., Andersson et al., 2014; Mohr et al., 2019). Moreover, computerized psychotherapy (with and without therapist support) has been found to be acceptable to clients (Andrews et al., 2010).

While stepped care and technology may alleviate the burden on mental healthcare systems, to ensure resources are used effectively, they need to be grounded in evidence-based treatments, such as acceptance and commitment therapy (ACT). ACT has been found to be efficacious for treating a range of presenting conditions (A-Tjak et al., 2015) and anxiety disorders and OCD in particular (Arch et al., 2012; Twohig et al., 2018). Moreover, ACT has been successfully delivered in internet-based self-help formats (French et al., 2017), making it suitable for a stepped care approach.

Considering the promise of stepped care in terms of increasing efficiency of finite resources and accessibility to evidence-based treatment, the aim of this pilot study was to evaluate the utility of providing an online self-help program to clients before they start therapy in a quasi-stepped care design. We tested the preliminary feasibility, acceptability, and 
effectiveness of using an online self-help ACT program as an initial step prior to in-person therapy among clients seeking treatment at an outpatient university clinic.

We predicted that (1) an initial self-help step would be acceptable (i.e., participants would adhere to the online program and report being satisfied with this first step); (2) this initial step would not adversely affect subsequent therapy acceptability (i.e., participants would still attend subsequent therapy if they needed it); (3) participants would significantly improve on mental health indices after the self-help step; and (4) participants would still show subsequent improvements from in-person therapy.

\section{Method}

\section{Participants}

Recruitment took place at an outpatient university community clinic in the western U.S., which typically has a waitlist of 2 weeks to 3 months. People who contacted the clinic to schedule an intake appointment were informed of the current study wherein they could access an online self-help program while they were on the waitlist. Participants who indicated interest in participating were referred to the research team for a phone screening.

Inclusion criteria were (1) people seeking treatment at the university community clinic (i.e., were on the clinic waitlist at the time of contact) who gave verbal consent to be contacted for the study, (2) ability to complete study measures and therapy in English (i.e., English fluency), and (3) at least 18 years old. There were no other inclusion criteria because we wanted to include a heterogeneous sample to evaluate the transdiagnostic utility of this adjunctive online self-help intervention. Exclusion criteria were (4) at least moderate suicidal risk as determined by a score of $\leq 8$ on the Mini International Neuropsychiatric Interview Suicidality module (described in Measures section below) and (5) no serious mental illness (i.e., bipolar disorder, psychosis) as 
determined by positive status on the respective Mini International Neuropsychiatric Interview diagnostic modules. Criteria (1) to (3) were confirmed through a phone screening. Participants interested in the study and who met criteria (1) to (3) were scheduled for an in-person intake during which the Mini International Neuropsychiatric Interview was conducted to confirm criteria (4) and (5). Eligible participants were invited to enroll in the study at the end of the intake meeting. In total, 51 participants met eligibility criteria (1) to (5) and completed the baseline assessment.

\section{Procedures}

Study procedures were approved by the university institutional review board. During the initial intake appointment, participants provided informed consent and a trained graduate research assistant conducted the Mini International Neuropsychiatric Interview, a semi-structured interview to assess for diagnoses and exclusion criteria (i.e., active suicidality, serious mental illness). Participants then completed study measures on a lab computer or iPad. At the end of the intake, participants completed an orientation for the online program during which they received instructions on how to log in to sessions and set up a schedule to complete two sessions a week.

Main assessments occurred at baseline (preprogram; Week 0), postprogram (after using the online program and before starting in-person therapy; Week 4), posttherapy (immediately after therapy termination; number of therapy sessions ranged from 1 to 21 ), and one-month follow-up (four weeks after posttherapy). Measures administered include the Acceptance and Action Questionnaire-II, Believability of Anxious Feelings and Thoughts Questionnaire, Depression Anxiety Stress Scales, Outcome Questionnaire-45.2, Quality of Life Scale, and Valued Living Questionnaire (described in detail in the Measures section below). Participants also completed a subset of these measures, the Acceptance and Action Questionnaire-II, 
Believability of Anxious Feelings and Thoughts Questionnaire, and Depression Anxiety Stress Scales, approximately every two weeks throughout the study (unless a main assessment was scheduled), starting with the midprogram assessment (Week 2). We collected qualitative data from participants at the end of the online program and therapists at the end of therapy to identify areas of the online program that were perceived as more and less effective.

\section{Interventions}

Online program. This study involved two distinct interventions: an online self-help program, Life Toolbox, and in-person therapy-as-usual. Life Toolbox is a shortened eightsession version of ACT Guide (https://scce.usu.edu/services/act-guide/), a publicly available 12session online self-help program. Life Toolbox emphasized acceptance (Away Moves, Carrying It with You), defusion (Noticing Hooks, Stepping Back), values (Your Values, Finding Values) and committed action (Setting Goals, Making Commitments). Combinations of these online selfhelp sessions, typically involving a longer 12-session format, have been found to be effective in improving mental health and psychological inflexibility in multiple clinical trials (e.g., Levin et al., 2017; Levin et al., 2016).

Participants received a link to the Life Toolbox website during study intake. The sessions were hosted on a secure Qualtrics platform and required a valid study ID to prevent unauthorized access of the online program. Participants were instructed to use Life Toolbox over four weeks at a rate of two sessions per week prior to starting therapy and received weekly email reminders about completing the sessions. Participants were allowed to navigate through the modules however they wanted, though they were recommended to complete modules in the prescribed order. 
Participants completed the Life Toolbox online program while they were on the clinic waitlist and started therapy after at least four weeks (duration of the online program) regardless of whether they completed all sessions of the online program. The progression from a lowintensity intervention to a higher-intensity intervention simulates a stepped care sequence, however, because we did not triage participants to steps of care based on clinical assessment (they were free to opt in or out of any part of treatment), we considered this a quasi-stepped care design.

Therapy. In-person therapy was conducted by seven clinical/counseling psychology Ph.D. graduate students in an ACT practicum over four semesters. Student therapists had 0-2 years of experience using ACT at the time of the study and received weekly supervision from a clinical psychologist with 20 years of experience delivering and researching ACT. Because the primary focus was not on evaluating the effects of in-person therapy, we did not collect data on ACT fidelity, but sessions were regularly viewed by the supervisor and weekly guidance to adhere to the ACT model was provided.

\section{Measures}

\section{Mini International Neuropsychiatric Interview Version 7.0.0 (Sheehan et al., 1998).}

The Mini International Neuropsychiatric Interview is a semi-structured diagnostic interview for DSM-5 disorders including generalized anxiety disorder, social anxiety disorder, and major depressive disorder. The Mini International Neuropsychiatric Interview has shown moderate agreement with clinical diagnoses (Verhoeven et al., 2017).

Acceptance and Action Questionnaire-II (Bond et al., 2011). The Acceptance and Action Questionnaire-II is a seven-item measure of psychological inflexibility. Items are rated from 1 (never true) to 7 (always true). The Acceptance and Action Questionnaire-II has shown 
good internal consistency, good test-retest reliability, criterion validity, and convergent validity (Bond et al., 2011). Internal reliability in the study was good $(\alpha=.87)$.

\section{Believability of Anxious Feelings and Thoughts Questionnaire (Herzberg et al.,}

2012). The Believability of Anxious Feelings and Thoughts Questionnaire is a 16-item measure of believability of thoughts related to anxiety or cognitive fusion. Items are rated from 1 (not at all believable) to 7 (completely believable). Higher scores indicate more cognitive fusion. The Believability of Anxious Feelings and Thoughts Questionnaire has shown excellent internal consistency, good test-retest reliability, and construct validity in nonclinical and clinical samples (Herzberg et al., 2012). Internal reliability in this study was good $(\alpha=.89)$.

Depression Anxiety Stress Scales (Henry \& Crawford, 2005). The Depression Anxiety Stress Scales is a 21-item self-report measure of overall psychological distress in the areas of depression, anxiety, and stress. Each area consists of seven items rated from 0 (never) to 3 (almost always). Higher scores reflect more psychological distress. The Depression Anxiety Stress Scales has demonstrated excellent psychometric properties (Henry \& Crawford, 2005). It showed excellent internal consistency in the current sample $(\alpha=.91)$.

Outcome Questionnaire-45.2 (Lambert et al., 1996). The Outcome Questionnaire-45.2 is a 45-item self-report measure of symptom severity and functional impairment. The Outcome Questionnaire-45.2 consists of three subscales: (a) symptom distress (i.e., symptoms related to anxiety, affective, and adjustment disorders); (b) interpersonal relations (i.e., loneliness, conflicts with others, dissatisfaction with quality of intimate relationships); and (c) social role (i.e., distress, inefficiency, and difficulties in social roles), Items are rated from 0 (never) to 4 (almost always). Higher scores indicate more symptom impairment. The Outcome Questionnaire-45.2 
has shown excellent psychometric properties (Lambert et al., 1996). It had excellent internal consistency in our sample $(\alpha=.93)$.

Quality of Life Scale (Burckhardt et al., 1989). The Quality of Life Scale is a 16-item self-report measure of general life functioning across broad life domains (e.g., relationships, employment, health, recreation). Items are rated from 1 (terrible) to 7 (delighted). Higher scores indicate better quality of life. The Quality of Life Scale has demonstrated good psychometric properties in multiple studies (Burckhardt \& Anderson, 2003; Burckhardt et al., 1989). It had good internal consistency in the current sample $(\alpha=.86)$.

Valued Living Questionnaire (Wilson et al., 2010). The Valued Living Questionnaire asks respondents to rate the importance and consistency of actions with values in 10 life domains including family, work, and physical self-care. Items are rated from 1 (not at all important/not at all consistent with my value) to 10 (extremely important/completely consistent with my value). Importance and Consistency scores are multiplied then averaged to produce a valued living composite; a higher score indicates more valued living. The scale has shown convergent validity (Wilson et al., 2010); internal consistency is less theoretically relevant because people may not value or engage in valued action consistently across different life domains.

Treatment Evaluation Inventory—Short Form (TEI-SF; Kelley et al., 1989). The Treatment Evaluation Inventory—Short Form is a nine-item measure of intervention acceptability (Kelley et al., 1989). We used a seven-item version of the Treatment Evaluation Inventory-Short Form; two items were omitted as they were not applicable to an adult sample. Each item is scored from 1 (strongly disagree) to 5 (strongly agree); higher scores indicate greater treatment acceptability (Kelley et al., 1989). The measure has good internal consistency and was sensitive to differences between treatments in previous research (Kelley et al., 1989). 
Internal consistency was good in our sample $(\alpha=.83)$. The Treatment Evaluation InventoryShort Form was administered at midprogram to minimize confounding effects of online program efficacy.

Satisfaction, Usability, and Perceived Effectiveness Rating Scale for Clients. The 25item Satisfaction, Usability, and Perceived Effectiveness Rating Scale for Clients was created for the present study to assess acceptability of the Life Toolbox online program. It was completed at postprogram. It includes six Satisfaction items (e.g., "Overall, I was satisfied with the quality of Life Toolbox"), 10 Usability items (e.g., "I thought Life Toolbox was easy to use”), and 9 Perceived Effectiveness items (e.g., "I felt that the skills taught in Life Toolbox were easy to understand"). Items are rated from 1 (strongly disagree) to 6 (strongly agree). Internal consistency was excellent $(\alpha=.95)$. The Satisfaction, Usability, and Perceived Effectiveness Rating Scale for Clients also has four open-ended prompts on reasons for not using Life Toolbox as scheduled (i.e., two sessions per week), most liked features, least liked features, and feedback for improving the online program.

Therapist feedback. Therapists answered three open-ended questions after therapy termination: what their clients found helpful about the online program, what their clients found unhelpful about the online program, and suggestions for improving the online program.

\section{Statistical Analyses}

Analyses were conducted with R version 3.6.3 (R Core Team, 2020) in RStudio (RStudio Team, 2020) using the following packages: tidyverse (Wickham, 2017), lme4 (Bates et al., 2015), texreg (Leifeld, 2013), psych (Revelle, 2018), effsize (Torchiano, 2017), and furniture (Barrett \& Brignone, 2017). 
Intervention feasibility and acceptability. Rates of online program completion and therapy initiation were used to assess the feasibility and acceptability of the quasi-stepped care intervention used in our study as demonstrated by participant engagement. Online program acceptability was evaluated quantitively using self-report scores on the Treatment Evaluation Inventory-Short Form and Satisfaction, Usability, and Perceived Effectiveness Rating Scale for Clients and qualitatively based on feedback provided by participants at postprogram and therapists at posttherapy.

Clinically significant and reliable change. Clinically significant change was indicated by scores that fell within two standard deviations of a normative mean (Jacobson \& Truax, 1991). Based on this criterion, we used the following cutoffs: $<26$ for the Acceptance and Action Questionnaire-II (Bond et al., 2011), <84 for the Believability of Anxious Feelings and Thoughts Questionnaire (Herzberg et al., 2012), < 29 for the Depression Anxiety Stress Scales (Henry \& Crawford, 2005), and < 87 for the Outcome Questionnaire-45.2 (Lambert et al., 1996). A reliable change index was calculated by dividing the difference between pretest and posttest scores by the standard error of the difference between the two scores. Reliable change was defined by a reliable change index greater than 1.96 (Jacobson \& Truax, 1991).

Progression through steps of care. We calculated the rates of reliable change through the online program and subsequent therapy for hypothesized processes of change (Acceptance and Action Questionnaire-II, Believability of Anxious Feelings and Thoughts Questionnaire), distress (Depression Anxiety Stress Scales), and symptom impairment (Outcome Questionnaire45.2) among participants who completed the posttherapy assessment $(n=17)$. The reliable change index was used as it represents magnitude of change rather than meeting a static cutoff. It is generally a stricter criterion for improvement than clinically significant change. These figures 
were used to determine the frequency of participants who (1) improved in therapy after they showed reliable gains from the online program, (2) only experienced gains in therapy but not from the online program, (3) only showed reliable change from the online program, and (4) did not show reliable change from either intervention.

Multilevel analyses. Multilevel or mixed-effects models were used to evaluate withingroup changes in outcomes over time using intent-to-treat analyses. We examined changes from (1) preprogram to postprogram (effect of online program), (2) pretherapy to posttherapy (effect of therapy), and (3) baseline to one-month follow-up (effect of online program plus therapy).

A series of nested models was tested for each outcome. For the Acceptance and Action Questionnaire-II, Believability of Anxious Feelings and Thoughts Questionnaire, and Depression Anxiety Stress Scales, which were administered at every assessment point, three models were specified. The first model was a null model specifying only random intercepts for participants. The second model included random slopes for participants, and the third model included a fixed effect of time. For the Outcome Questionnaire-45.2, Valued Living Questionnaire, and Quality of Life Scale, which were only given at major assessment points, the second model specifying random slopes was omitted due to insufficient observations over time (random effects parameters and residual variance were unidentifiable). Thus, only the null and time as a fixed effect models were tested.

In each model comparison, we used likelihood ratio tests to determine the best-fitting model at $p<.05$. Final models were estimated using the maximum likelihood criterion or an optimizer in cases where models did not converge. Only coefficient estimates from the bestfitting models are reported and coefficient $p$-values are based on the Satterthwaite approximation 
to degrees of freedom. We calculated Hedges' $g$ effect sizes for all outcomes; 0.2 corresponds to a small effect, 0.5 to a medium effect, and 0.8 to a large effect.

\section{Results}

\section{Participants and Attrition}

Fifty-four participants met initial screening criteria, but three were ultimately excluded due to active suicidality as determined by the Mini International Neuropsychiatric Interview. The remaining 51 participants were included in our intent-to-treat analyses. Of the 51 participants who started the program, 34 participants completed the postprogram assessment $(33.3 \%$ attrition). Among the 28 participants who started therapy, 17 completed the posttherapy assessment (39.3\% attrition) and 25 completed the follow-up assessment (10.7\% attrition; see Figure 1).

The mean age of our sample was 22.9 years $(\mathrm{SD}=5.0)$. Approximately half the sample identified as female. Most participants identified as White, heterosexual, and members of The Church of Jesus Christ of Latter-day Saints (see Table 1 for more details). The most common diagnoses in our sample based on the Mini International Neuropsychiatric Interview were major depressive disorder (49.0\%), generalized anxiety disorder (45.1\%), and panic disorder (37.3\%). Sixty-three percent of participants were assigned more than one diagnosis.

\section{Intervention Feasibility and Acceptability}

Online program completion and therapy initiation. Figure 1 provides a flowchart of online program progress and study retention. Of the 51 participants who completed the baseline assessment, $72.5 \%$ completed at least one online program session, $49.0 \%$ completed at least half of the online program (four sessions), and $25.5 \%$ completed the entire online program (eight sessions). The mean number of online program sessions completed was $3.7(\mathrm{SD}=3.2)$. 
Among the 37 participants who started the online program, $73.0 \%$ started in-person therapy (one participant who attended in-person therapy did not start the online program). The mean number of online sessions completed was $5.1(\mathrm{SD}=2.7)$ in the subset who started inperson therapy. Among the 13 who completed the entire online program, $84.6 \%$ started therapy.

Participants who started $\left(\chi^{2}=15.22, p<.001\right)$ or finished the online program $\left(\chi^{2}=4.72\right.$, $p=.030$ ) were more likely to start therapy than those who did not. The mean number of therapy sessions attended was $7.5(\mathrm{SD}=5.4$, range $=1$ to 21$)$.

Self-report measures. The mean Treatment Evaluation Inventory-Short Form score was $26.8(\mathrm{SD}=4.4)$, indicating good acceptability for the online program based on established cutoffs (21 indicates moderate acceptability). The mean Satisfaction, Usability, and Perceived Effectiveness Rating Scale for Clients score was $111(\mathrm{SD}=20.5)$ out of 150 , indicating that user satisfaction with, perceived usability of, and perceived effectiveness of the online program were high.

\section{Qualitative feedback.}

Participants. Responses to the three open-ended questions on most liked and least liked features of the online program and feedback on improving the online program were coded for themes. In the following results, multiple reasons might have been reported by the same participant (i.e., there may be double counting in the $n$ s).

Participants liked that sessions were brief and easy to use $(n=15)$, learning specific tools relevant to their struggles $(n=7)$, the use of metaphors $(n=6)$, that they were able to use the modules based on their needs (e.g., pacing, intervention focus; $n=5$ ), the psychoeducation components $(n=4)$, and that sessions prepared them for therapy $(n=3)$. 
Participants did not like that the sessions were not specific to their needs and concerns ( $n$ $=5)$, lacked face-to-face interaction $(n=2)$, had spelling and grammatical errors $(n=2)$, were too wordy $(n=1)$, did not teach anything new $(n=1)$, did not build on each other effectively $(n$ $=1$, were only useful in the short-term $(n=1)$, were incompatible with mobile devices $(n=1)$, and did not provide accountability $(n=1)$.

Reasons for not using the modules as scheduled included forgetting to do them $(n=14)$, lack of time $(n=14)$, lack of interest in the online program $(n=3)$, not perceiving the online program as helpful $(n=3)$, preference for in-person therapy $(n=2)$, difficulty of use $(n=1)$, and discomfort using the online program in front of others $(n=1)$. No one reported accessibility or technical issues.

Regarding areas for improvement, participants suggested providing more support in conjunction with online program use (e.g., coaching, therapy; $n=3)$, proofreading the content $(n$ $=3)$, increasing interactive features $(n=1)$, developing an app version of the online program $(n=$ $1)$, and giving a clearer description of context of use at the outset $(n=1)$.

Therapists. Therapists reported that for clients who had used the online program, benefits included having a headstart in therapy (e.g., clients already knew the skills and vocabulary; $n=$ 14) and being able to leverage the app to facilitate learning $(n=1)$. A therapist reported that one participant chose not to start therapy as they were already effectively using acceptance-based strategies at intake. The only drawback noted from a therapist was that the online program might have dampened one participant's interest in ACT as they did not find the online program sessions helpful. In addition, therapists commonly observed that incompletion of the online program was a greater barrier than issues with the online program itself. Therapists reported that the online program either decreased $(n=6)$ or had no effect $(n=5)$ on therapist burden. 
For areas for improvement, therapists suggested finding ways to increase participants' engagement with the online program $(n=6)$ and providing therapists with guidance on how to effectively integrate online program content into therapy sessions $(n=3)$.

\section{Clinically Significant and Reliable Change}

Preprogram to postprogram. Most participants (75.9 to $79.3 \%$ ) showed clinically significant change for cognitive fusion, psychological distress, and symptom impairment and reliable change for cognitive fusion $(63.0 \%)$ and symptom impairment $(100 \%)$ by the end of the online program period, indicating that the online program led to improvement in most outcomes, especially cognitive fusion and symptom impairment (see Table 4).

Pretherapy to posttherapy. Most participants showed clinically significant (64.7 to $100 \%)$ and reliable changes (56.3 to $100 \%$ ) for psychological inflexibility, cognitive fusion, psychological distress, and symptom impairment by the end of therapy, indicating that therapy had an incremental positive effect on these outcomes (see Table 4).

Preprogram (baseline) to one-month follow-up. Most participants showed clinically significant (76.0 to $100 \%)$ and reliable (60.0 to $91.7 \%)$ changes in all outcomes tested by followup, suggesting that the online program-to-therapy sequence was effective (see Table 4).

\section{Progression Through Steps of Care}

The following findings only represent participants who completed the posttherapy assessment $(n=17)$. Those who reliably improved in each intervention (i.e., benefited from therapy after they had experienced reliable gains from the online program) ranged from 5.6 to $100 \%$ across outcomes. In particular, the rate was $100 \%$ for symptom impairment, which means, if we take symptom impairment as the outcome of interest, all participants appropriately progressed through the quasi-stepped care model (i.e., received care as needed). The percentage 
of participants who only experienced gains in therapy or who benefited from being stepped up to in-person therapy ranged from 26.7 to $62.5 \%$; this group might have needed to start therapy sooner. Participants who benefited from neither intervention ( 0 to $22.2 \%$ ) or who benefited from the online program but not therapy (potentially those who may not have needed further treatment but still opted in to therapy; 0 to $16.7 \%$ ) were less common.

\section{Changes in Outcomes Over Time}

Coefficient estimates and $95 \%$ confidence intervals for best-fitting multilevel models are presented in Table 2. Means, standard deviations, and effect sizes over the course of the study are reported in Table 3.

Preprogram to postprogram. Over the course of the online program, we observed small to medium decreases in psychological inflexibility, cognitive fusion, and symptom impairment (absolute Hedges' $g=0.36$ to 0.67 ) and no significant changes in distress, quality of life, or valued living (absolute Hedges' $g=0.02$ to 0.29 ).

Pretherapy to posttherapy. Over the course of therapy, we found medium to large decreases in psychological inflexibility, cognitive fusion, and symptom impairment (absolute Hedges' $g=0.43$ to 0.99 ) and no significant changes in distress, quality of life, or valued living (absolute Hedges' $g=0.23$ to 0.45 ).

Preprogram (baseline) to one-month follow-up. Over the course of the study, we found medium to large improvements in psychological inflexibility, cognitive fusion, distress, symptom impairment, and quality of life (absolute Hedges' $g=0.47$ to 2.04 ) and no significant change in valued living (Hedges' $g=0.19$ ).

\section{Discussion}


In the present study, we examined the feasibility, acceptability, and effectiveness of providing an online self-help ACT program to clients on the waitlist at an outpatient clinic prior to in-person therapy. The objective was to evaluate the utility of implementing an easily accessible online self-help program in a quasi-stepped care model and determine if this approach may be a viable way to reduce client and therapist burden without compromising quality of care. Unlike the current study, previous studies examined the effects of web-based self-help alone (e.g., Buntrock et al., 2016), a stepped care model that still involved therapist contact at the outset (e.g., Nordgreen et al., 2016), or "blended" therapy in which online modules could be completed before, during, or after face-to-face therapy (Kenter et al., 2015). However, we tested the incremental benefit of engaging in web-based self-help while on a clinic waitlist before seeing a therapist without adding to therapist's existing clinical responsibilities.

With respect to online program engagement, completion rates were adequate, partially consistent with our prediction. Approximately three-quarters of our sample started the online program, approximately half completed at least four sessions, and approximately a quarter completed all eight sessions. Among those who started the online program, the mean number of sessions was 5.1, indicating participants completed more than $50 \%$ of the online program on average once they started it. These results suggest moderate acceptability of the online program, which is consistent with self-reported treatment acceptability.

Approximately a quarter of participants did not start the online program at all, and another quarter did not complete at least half of the online program; this subset represents those with poor online program engagement. The relatively high dropout rate may be a function of the quasi-stepped care design (Nordgreen et al., 2016), limited ability of the online program to engage users, and/or inappropriate matching of services to client needs and characteristics. For 
example, five participants reported that the sessions were not specific enough to their struggles. These findings highlight the importance of assigning clients to the appropriate level of care, balancing client needs and preferences with available resources. Overcorrection in either direction (e.g., providing therapy to clients who would sufficiently benefit from a lower intensity intervention or giving a self-help program to clients who urgently need in-person care) may lead to poorer outcomes overall, inefficient use of resources, and even mistrust of the mental healthcare system that discourages subsequent treatment seeking.

Future research should examine how client variables moderate treatment response to unguided self-help internet interventions. For example, a study testing an unguided online intervention for depression found that participants who reported lower willingness to change and higher education were more likely to experience increased depressive symptoms compared to treatment-as-usual (Lüdtke et al., 2018). While this study did not identify moderators of online program acceptability and effectiveness, identifying these variables would clarify how to triage clients and may provide actuarial guidance for clinical decision making.

We used two broad metrics to evaluate online program effectiveness: (1) clinically significant change and reliable change (yes/no binary outcome) and (2) significant changes in scores over time (continuous aggregate outcome). Both metrics are reported in order below and provide slightly different perspectives of online program effectiveness.

Following the online program, we observed clinically significant change rates of 75.9 to 79.3\% for cognitive fusion, distress, and symptom impairment and reliable change rates of 31.0 to $100 \%$ for psychological inflexibility, cognitive fusion, and distress, showing that an initial lower intensity treatment step, like Life Toolbox, led to meaningful change for a sizable portion of participants. Of note, these figures include all participants who completed the postprogram 
assessment, including those who did not complete the entire online program. Thus, they represent a more conservative estimate of the effectiveness of the online program. More precisely, these numbers reflect the effect of being offered access to the online program rather than completing the online program per se.

For participants who started treatment, 60 to $100 \%$ reliably improved from the online program and again from subsequent treatment on three of four outcomes, indicating that most of our sample experienced reliable change from each step of care. Participants who started or completed the online program were also more likely to enroll in therapy afterward, suggesting that use of the online program did not affect subsequent therapy acceptability, as predicted. Moreover, therapists overwhelmingly reported that therapy proceeded more quickly when clients had already used the online program and been exposed to basic ACT concepts, suggesting that the online program may have cascading effects on efficiency by potentially reducing the number of therapy sessions needed.

With respect to aggregate continuous outcomes (i.e., averaging across participants), we observed decreases in psychological inflexibility, cognitive fusion, and symptom impairment but no changes in distress, quality of life, and valued living over the course of the online program alone. This pattern was replicated over the course of treatment alone: psychological inflexibility, cognitive fusion, and symptom impairment further decreased but there were no changes in distress, quality of life, and valued living. These results indicate that, individually, the online program and therapy improved hypothesized processes of change and symptoms but were less effective at changing distress, quality of life, and valued living. However, we found reductions in distress and quality of life over the entire study (online program plus therapy), suggesting that these outcomes were only affected by the interventions cumulatively. Thus, it seems that there 
was incremental benefit to completing the online program prior to starting in-person therapy and from continuing with therapy after completing the online program.

Overall, our results tentatively support offering an online self-help resource to people who do not have immediate access to in-person therapy. Participants who used the online program were more likely to start therapy and most participants who completed both interventions generally benefited from each of them. In particular, given the option between no treatment and self-help, participants who self-select for such self-help online programs may find them useful. Thus, lower intensity interventions like online self-help programs may be a feasible and acceptable way to reduce therapist burden in outpatient clinics for certain types of clients - by initiating therapeutic progress even before intake and serving as a starting point for people seeking treatment within a stepped care model.

\section{Suggestions for Refining the Intervention}

Despite generally encouraging results from this pilot trial, we did not observe any meaningful change in valued living. To the extent that ACT emphasizes living consistently with values as a metric of treatment success, the lack of change in valued living reveals limitations of the tested interventions and suggests that the online program needs better ways to target valued action. For example, because the lack of accountability inherent in self-help interventions may make instituting behavioral changes harder, therapy following the online program could focus on reinforcing any changes made during the online program. At the same time, changes in valued action could lag relative to other outcomes given that implementing sustained behavioral change through forming new habits may require more time. Alternatively, the Valued Living Questionnaire may not have been sufficiently sensitive to changes in valued behavior. 
In addition, the current self-help online intervention was associated with lower effect sizes than those reported for another unguided internet-based treatment (Morgan et al., 2017) and might have been beset with similar limitations to those discussed for unguided iCBT in general: high attrition, small effects relative to guided internet-based and face-to-face interventions, and potential selection bias (i.e., participants who benefit more from self-help interventions may be more motivated to begin with; Karyotaki et al., 2017). Our high attrition rate underscores the need to pair evidence-based treatment options with evidence-based triaging and to develop lowburden resources that sufficiently engage clients who were originally seeking in-person therapy.

These findings also highlight the real-world challenges of implementing online programs in clinical settings without significant buy-in from clinic staff. It is possible that adding therapist support, as suggested by several participants, would bolster efficacy of the online program (Karyotaki et al., 2021), such that the benefit of increasing therapist responsibilities may outweigh the cost. Ultimately, to maximize efficiency and effectiveness in a stepped care system, we need to determine how to match clients to the appropriate step of care, make evidence-based treatment available and accessible at each step, and increase the reach of these interventions by minimizing attrition (e.g., making self-help resources more interactive).

\section{Limitations}

First, because of the nature of research participation, our sequencing of the interventions was not a true stepped care model in the sense that participants self-selected into in-person therapy rather than were identified by therapists as requiring in-person therapy. As such, we did not match participants to initial level of care or change level of care based on their presentation, which is the crux of the stepped care model. Nonetheless, we did test a quasi-stepped care sequence that may inform the design of future stepped care studies (e.g., using an online self- 
help program as we did but adding matching elements). Furthermore, this form of implementation may be even more acceptable to clients since they have the autonomy to decide what their treatment looks like. Second, our sample was relatively homogeneous, precluding generalization to different populations. While preliminary tests of effectiveness can guide us toward which interventions to pursue or modify, future tests need to include diverse samples to ascertain what or if any cultural adaptions are needed to replicate positive treatment effects. Finally, we did not have a control group in this open trial design, so we could not determine if the effects observed were superior to treatment-as-usual. However, the gestalt effects of both interventions on psychological distress and quality of life provide some support for the utility of the online program in addition to in-person therapy. Studies with greater experimental control are needed to ascertain the incremental benefit of accessing a self-help online program prior to inperson therapy. 


\section{References}

A-Tjak, J. G. L., Davis, M. L., Morina, N., Powers, M. B., Smits, J. A., \& Emmelkamp, P. M. (2015). A meta-analysis of the efficacy of acceptance and commitment therapy for clinically relevant mental and physical health problems. Psychotherapy and Psychosomatics, 84(1), 30-36. https://doi.org/10.1159/000365764

Andersson, G., Cuijpers, P., Carlbring, P., Riper, H., \& Hedman, E. (2014, 2014/10/01). Guided Internet-based vs. face-to-face cognitive behavior therapy for psychiatric and somatic disorders: A systematic review and meta-analysis. World Psychiatry, 13(3), 288-295. https://doi.org/10.1002/wps.20151

Andrews, G., Cuijpers, P., Craske, M. G., McEvoy, P., \& Titov, N. (2010, Oct 13). Computer therapy for the anxiety and depressive disorders is effective, acceptable and practical health care: A meta-analysis. PLoS One, 5(10), e13196. https://doi.org/10.1371/journal.pone.0013196

Arch, J. J., Eifert, G. H., Davies, C., Vilardaga, J. C. P., Rose, R. D., \& Craske, M. G. (2012). Randomized clinical trial of cognitive behavioral therapy (CBT) versus acceptance and commitment therapy (ACT) for mixed anxiety disorders. Journal of Consulting and Clinical Psychology, 80(5), 750-765. https://doi.org/10.1037/a0028310

Barrett, T. S., \& Brignone, E. (2017). Furniture for quantitative scientists. $R$ Journal, 9, 142-148.

Bates, D., Maechler, M., Bolker, B., \& Walker, S. (2015). Fitting linear mixed-effects models using lme4. Journal of Statistical Software, 67(1), 1-48. https://doi.org/10.18637/jss.v067.i01

Bond, F. W., Hayes, S. C., Baer, R. A., Carpenter, K. M., Guenole, N., Orcutt, H. K., Waltz, T., \& Zettle, R. D. (2011). Preliminary psychometric properties of the Acceptance and Action Questionnaire-II: A revised measure of psychological inflexibility and experiential avoidance. Behavior Therapy, 42(4), 676-688. https://doi.org/10.1016/j.beth.2011.03.007

Bower, P., \& Gilbody, S. (2005). Stepped care in psychological therapies: Access, effectiveness and efficiency: Narrative literature review. British Journal of Psychiatry, 186(1), 11-17. https://doi.org/10.1192/bjp.186.1.11

Buntrock, C., Ebert, D. D., Lehr, D., Smit, F., Riper, H., Berking, M., \& Cuijpers, P. (2016). Effect of a web-based guided self-help intervention for prevention of major depression in adults with subthreshold depression: A randomized clinical trial. JAMA, 315(17), 18541863. https://doi.org/10.1001/jama.2016.4326

Burckhardt, C. S., \& Anderson, K. L. (2003). The Quality of Life Scale (QOLS): Reliability, validity, and utilization. Health and Quality of Life Outcomes, 1(60). https://doi.org/10.1186/1477-7525-1-60 
Burckhardt, C. S., Woods, S. L., Schultz, A. A., \& Ziebarth, D. M. (1989). Quality of life of adults with chronic illness: A psychometric study. Research in Nursing \& Health, 12(6), 347-354. https://doi.org/10.1002/nur.4770120604

French, K., Golijani-Moghaddam, N., \& Schröder, T. (2017). What is the evidence for the efficacy of self-help acceptance and commitment therapy? A systematic review and meta-analysis. Journal of Contextual Behavioral Science. https://doi.org/10.1016/j.jcbs.2017.08.002

Henry, J. D., \& Crawford, J. R. (2005, Jun). The short-form version of the Depression Anxiety Stress Scales (DASS-21): construct validity and normative data in a large non-clinical sample. British Journal of Clinical Psychology, 44(Pt 2), 227-239. https://doi.org/10.1348/014466505X29657

Herzberg, K. N., Sheppard, S. C., Forsyth, J. P., Credé, M., Earleywine, M., \& Eifert, G. H. (2012). The Believability of Anxious Feelings and Thoughts Questionnaire (BAFT): A psychometric evaluation of cognitive fusion in a nonclinical and highly anxious community sample. Psychological Assessment, 24(4), 877-891. https://doi.org/10.1037/a0027782

Ho, F. Y.-Y., Yeung, W.-F., Ng, T. H.-Y., \& Chan, C. S. (2016, 2016/07/05). The efficacy and cost-effectiveness of stepped care prevention and treatment for depressive and/or anxiety disorders: A systematic review and meta-analysis. Scientific Reports, 6(1), 29281. https://doi.org/10.1038/srep29281

Jacobson, N. S., \& Truax, P. (1991). Clinical significance: A statistical approach to defining meaningful change in psychotherapy research. Journal of Counsulting and Clinical Psychology, 59(1), 12-19. https://doi.org/10.1037/0022-006X.59.1.12

Karyotaki, E., Efthimiou, O., Miguel, C., Bermpohl, F. M. g., Furukawa, T. A., Cuijpers, P., \& Individual Patient Data Meta-Analyses for Depression Collaboration. (2021). Internetbased cognitive behavioral therapy for depression: A systematic review and individual patient data network meta-analysis. JAMA psychiatry, 78(4), 361-371. https://doi.org/10.1001/jamapsychiatry.2020.4364

Karyotaki, E., Riper, H., Twisk, J., Hoogendoorn, A., Kleiboer, A., Mira, A., Mackinnon, A., Meyer, B., Botella, C., Littlewood, E., Andersson, G., Christensen, H., Klein, J. P., Schröder, J., Bretón-López, J., Scheider, J., Griffiths, K., Farrer, L., Huibers, M. J. H., Phillips, R., Gilbody, S., Moritz, S., Berger, T., Pop, V., Spek, V., \& Cuijpers, P. (2017). Efficacy of self-guided internet-based cognitive behavioral therapy in the treatment of depressive symptoms: A meta-analysis of individual participant data. JAMA psychiatry, 74(4), 351-359. https://doi.org/10.1001/jamapsychiatry.2017.0044 
Kelley, M. L., Heffer, R. W., Gresham, F. M., \& Elliot, S. N. (1989). Development of a modified Treatment Evaluation Inventory. Journal of Psychopathology and Behavioral Assessment, 11(3), 235-247. https://doi.org/10.1007/BF00960495

Kenter, R. M. F., van de Ven, P. M., Cuijpers, P., Koole, G., Niamat, S., Gerrits, R. S., Willems, M., \& van Straten, A. (2015). Costs and effects of Internet cognitive behavioral treatment blended with face-to-face treatment: Results from a naturalistic study. Internet Interventions, 2(1), 77-83. https://doi.org/10.1016/j.invent.2015.01.001

Kessler, R. C., Angermeyer, M., Anthony, J. C., De Graaf, R., Demyttenaere, K., Gasquet, I., De Girolamo, G., Gluzman, S., Gureje, O., \& Haro, J. M. (2007). Lifetime prevalence and age-of-onset distributions of mental disorders in the World Health Organization's World Mental Health Survey Initiative. World Psychiatry, 6(3), 168.

Kessler, R. C., Petukhova, M., Sampson, N. A., Zaslavsky, A. M., \& Wittchen, H. (2012, 2012). Twelve-month and lifetime prevalence and lifetime morbid risk of anxiety and mood disorders in the United States. International Journal of Methods in Psychiatric Research, 21(3), 169-184. https://doi.org/10.1002/mpr.1359

Lambert, M. J., Burlingame, G. M., Umphress, V., Hansen, N. B., Vermeersch, D. A., Clouse, G. C., \& Yanchar, S. C. (1996). The reliability and validity of the Outcome Questionnaire. Clinical Psychology \& Psychotherapy, 3(4), 249-258. https://doi.org/10.1002/(SICI)1099-0879(199612)3:4<249::AID-CPP106>3.0.CO;2-S

Leifeld, P. (2013). texreg: Conversion of statistical model output in R to LaTeX and HTML tables. Journal of Statistical Software, 55(8), 1-24. https://doi.org/10.18637/jss.v055.i08

Levin, M. E., Haeger, J. A., Pierce, B. G., \& Twohig, M. P. (2017). Web-based acceptance and commitment therapy for mental health problems in college students: A randomized controlled trial. Behavior Modification, 41(1), 141-162. https://doi.org/10.1177/0145445516659645.

Levin, M. E., Hayes, S. C., Pistorello, J., \& Seeley, J. R. (2016). Web-based self-help for preventing mental health problems in universities: Comparing acceptance and commitment training to mental health education. Journal of Clinical Psychology, 72(3), 207-225. https://doi.org/10.1002/jclp.22254.

Lüdtke, T., Westermann, S., Pult, L. K., Schneider, B. C., Pfuhl, G., \& Moritz, S. (2018). Evaluation of a brief unguided psychological online intervention for depression: A controlled trial including exploratory moderator analyses. Internet Interventions, 13, 7381. https://doi.org/10.1016/j.invent.2018.06.004

Matcham, F., Rayner, L., Hutton, J., Monk, A., Steel, C., \& Hotopf, M. (2014). Self-help interventions for symptoms of depression, anxiety and psychological distress in patients with physical illnesses: A systematic review and meta-analysis. Clinical Psychology Review, 34(2), 141-157. https://doi.org/10.1016/j.cpr.2014.01.005 
Mohr, D. C., Lattie, E. G., Tomasino, K. N. h., Kwasny, M. J., Kaiser, S. M., Gray, E. L., Alam, N., Jordan, N., \& Schueller, S. M. (2019, 2019/12/01/). A randomized noninferiority trial evaluating remotely-delivered stepped care for depression using internet cognitive behavioral therapy (CBT) and telephone CBT. Behaviour Research and Therapy, 123, 103485. https://doi.org/https://doi.org/10.1016/j.brat.2019.103485

Morgan, C., Mason, E., Newby, J. M., Mahoney, A. E. J., Hobbs, M. J., McAloon, J., \& Andrews, G. (2017). The effectiveness of unguided internet cognitive behavioural therapy for mixed anxiety and depression. Internet Interventions, 10, 47-53. https://doi.org/10.1016/j.invent.2017.10.003

Nordgreen, T., Haug, T., Öst, L., Andersson, G., Carlbring, P., Kvale, G., Tangen, T., Heiervang, E., \& Havik, O. E. (2016, 2016/03/01/). Stepped care versus direct face-to-face cognitive behavior therapy for social anxiety disorder and panic disorder: A randomized effectiveness trial. Behavior Therapy, 47(2), 166-183. https://doi.org/https://doi.org/10.1016/j.beth.2015.10.004

R Core Team. (2020). R: A language and environment for statistical computing. R Foundation for Statistical Computing. https://www.R-project.org/

Revelle, W. (2018). psych: Procedures for psychological, psychometric, and personality research. $R$ package version 1.8.10. https://CRAN.R-project.org/package=psych

RStudio Team. (2020). RStudio: Integrated Development for R. RStudio, Inc. http://www.rstudio.com/

Seekles, W., van Straten, A., Beekman, A., van Marwijk, H., \& Cuijpers, P. (2011). Stepped care treatment for depression and anxiety in primary care. A randomized controlled trial. Trials, 12, 171-171. https://doi.org/10.1186/1745-6215-12-171

Sheehan, D. V., Lecrubier, Y., Sheehan, K. H., Amorim, P., Janavs, J., Weiller, E., Hergueta, T., Baker, R., \& Dunbar, G. C. (1998, 1998). The Mini-International Neuropsychiatric Interview (M.I.N.I.): The development and validation of a structured diagnostic psychiatric interview for DSM-IV and ICD-10. The Journal of Clinical Psychiatry, 59 (Suppl 20), 22-33. http://www.ncbi.nlm.nih.gov/pubmed/9881538

Torchiano, M. (2017). effsize: Efficient Effect Size Computation. R package version 0.7.1. https://CRAN.R-project.org/package=effsize

Twohig, M. P., Abramowitz, J. S., Smith, B. M., Fabricant, L. E., Jacoby, R. J., Morrison, K. L., Bluett, E. J., Reuman, L., Blakey, S. M., \& Lederman, T. (2018). Adding acceptance and commitment therapy to exposure and response prevention for obsessive-compulsive disorder: A randomized controlled trial. Behaviour Research and Therapy, 108, 1-9. https://doi.org/10.1016/j.brat.2018.06.005 
van Spijker, B. A. J., Majo, M. C., Smit, F., van Straten, A., \& Kerkhof, A. J. F. M. (2012, 2012/10/26). Reducing suicidal ideation: Cost-effectiveness analysis of a randomized controlled trial of unguided web-based self-help. Journal of Medical Internet Research, 14(5), e141. https://doi.org/10.2196/jmir.1966

van Straten, A., Cuijpers, P., \& Smits, N. (2008, 2008/3/25). Effectiveness of a web-based selfhelp intervention for symptoms of depression, anxiety, and stress: Randomized controlled trial. Journal of Medical Internet Research, 10(1), e7. https://doi.org/10.2196/jmir.954

van't Hof, E., Cuijpers, P., \& Stein, D. J. (2009). Self-help and internet-guided interventions in depression and anxiety disorders: A systematic review of meta-analyses. CNS Spectrums, 14(S3), 34-40. https://doi.org/10.1017/S1092852900027279

Verhoeven, F. E. A., Swaab, L., Carlier, I. V. E., van Hemert, A. M., Zitman, F. G., Ruhe, H. G., Schoevers, R. A., \& Giltay, E. J. (2017, Oct 15). Agreement between clinical and MINI diagnoses in outpatients with mood and anxiety disorders. Journal of Affective Disorders, 221, 268-274. https://doi.org/10.1016/j.jad.2017.06.041

Vigo, D., Thornicroft, G., \& Atun, R. (2016). Estimating the true global burden of mental illness. The Lancet Psychiatry, 3(2), 171-178.

Wickham, H. (2017). tidyverse: Easily install and load 'Tidyverse' packages. R package version 1.1.1. http://CRAN.R-project.org/package=tidyverse

Wilson, K. G., Sandoz, E. K., Kitchens, J., \& Roberts, M. (2010). The Valued Living Questionnaire: Defining and measuring valued action within a behavioral framework. The Psychological Record, 60, 249-272. https://doi.org/10.1007/BF03395706 
Table 1

Sample Description $(N=51)$

\begin{tabular}{|c|c|}
\hline & $\begin{array}{l}\text { Mean/Count } \\
(\mathrm{SD} / \%)\end{array}$ \\
\hline Age & $22.9(5.0)$ \\
\hline \multicolumn{2}{|l|}{ Gender identity } \\
\hline Female & $24(47.1 \%)$ \\
\hline Male & $26(51 \%)$ \\
\hline Not listed & $1(2 \%)$ \\
\hline \multicolumn{2}{|l|}{ Ethnicity } \\
\hline Black & $1(2 \%)$ \\
\hline Latinx/Hispanic & $3(5.9 \%)$ \\
\hline Multiracial & $2(3.9 \%)$ \\
\hline White & $44(86.3 \%)$ \\
\hline Not listed & $1(2 \%)$ \\
\hline \multicolumn{2}{|l|}{ Religious affiliation } \\
\hline Catholic & $3(5.9 \%)$ \\
\hline The Church of Jesus Christ of Latter-day Saints/Mormon & $29(56.9 \%)$ \\
\hline Not religious & $12(23.5 \%)$ \\
\hline Protestant & $3(5.9 \%)$ \\
\hline Not listed & $4(7.8 \%)$ \\
\hline \multicolumn{2}{|l|}{ Sexual orientation } \\
\hline Asexual & $3(5.9 \%)$ \\
\hline Bisexual & $5(9.8 \%)$ \\
\hline Gay or lesbian & $1(2 \%)$ \\
\hline Heterosexual & $41(80.4 \%)$ \\
\hline Not listed & $1(2 \%)$ \\
\hline \multicolumn{2}{|l|}{ Highest education level } \\
\hline Some graduate school & $4(7.8 \%)$ \\
\hline B.A./B.S. or equivalent & $7(13.7 \%)$ \\
\hline Associate degree & $9(17.6 \%)$ \\
\hline Some college & $24(47.1 \%)$ \\
\hline High school diploma or equivalent & $7(13.7 \%)$ \\
\hline \multicolumn{2}{|l|}{ Employment status } \\
\hline Part-time student & $5(9.8 \%)$ \\
\hline Full-time student & $27(52.9 \%)$ \\
\hline Working part-time & $6(11.8 \%)$ \\
\hline Working full-time & $5(9.8 \%)$ \\
\hline Unemployed/not working & $5(9.8 \%)$ \\
\hline Not listed & $3(5.9 \%)$ \\
\hline
\end{tabular}


Household income

\begin{tabular}{|c|c|}
\hline$\$ 0-\$ 15,000$ & $31(60.8 \%)$ \\
\hline$\$ 15,001-\$ 25,000$ & $10(19.6 \%)$ \\
\hline$\$ 25,001-\$ 35,000$ & $3(5.9 \%)$ \\
\hline$\$ 35,001-\$ 50,000$ & $2(3.9 \%)$ \\
\hline$\$ 50,001-\$ 75,000$ & $3(5.9 \%)$ \\
\hline$\$ 75,001-\$ 100,000$ & $1(2 \%)$ \\
\hline$\$ 100,001-\$ 200,000$ & $1(2 \%)$ \\
\hline \multicolumn{2}{|l|}{ Relationship status } \\
\hline Married & $11(21.6 \%)$ \\
\hline Single & $38(74.5 \%)$ \\
\hline Not listed & $2(3.9 \%)$ \\
\hline \multicolumn{2}{|l|}{ Previous therapy experience } \\
\hline Yes & $36(70.6 \%)$ \\
\hline No & $15(29.4 \%)$ \\
\hline \multicolumn{2}{|l|}{ Diagnosis $^{1}$} \\
\hline Generalized anxiety disorder & $23(45.1 \%)$ \\
\hline Obsessive-compulsive disorder & $11(21.6 \%)$ \\
\hline Social anxiety disorder & $10(19.6 \%)$ \\
\hline Panic disorder & $19(37.3 \%)$ \\
\hline Agoraphobia & $3(5.9 \%)$ \\
\hline Major depressive disorder & $25(49 \%)$ \\
\hline Premenstrual dysphoric disorder & $1(2 \%)$ \\
\hline No diagnosis & $1(2 \%)$ \\
\hline Other & $5(9.8 \%)$ \\
\hline
\end{tabular}

${ }^{1}$ Percentages do not add up to $100 \%$ due to co-existing diagnoses.

Note. Other diagnoses included alcohol use disorder $(n=1)$, binge eating disorder $(n=1)$, bulimia nervosa $(n=1)$, and posttraumatic stress disorder $(n=2)$. 
Table 2

Coefficient Estimates From Best-Fitting Multilevel Models

\begin{tabular}{|c|c|c|c|c|c|c|}
\hline & AAQ-II & BAFT & DASS-21 & OQ-45 & QOLS & VLQ \\
\hline & \multicolumn{6}{|c|}{ Preprogram to postprogram } \\
\hline Intercept & $\begin{array}{c}31.30[28.93 \\
33.67]^{*}\end{array}$ & $\begin{array}{l}77.77[73.55 \\
\quad 82.00]^{*}\end{array}$ & $\begin{array}{c}23.38[20.18 \\
26.57]^{*}\end{array}$ & $\begin{array}{c}73.86[67.22 \\
80.50]^{*}\end{array}$ & $\begin{array}{l}77.29[73.78 \\
80.79]^{*}\end{array}$ & $\begin{array}{c}50.40[46.51 \\
54.29]^{*}\end{array}$ \\
\hline Week & $\begin{array}{l}-0.79[-1.54 \\
-0.05]^{*}\end{array}$ & $\begin{array}{c}-3.53[-5.11 \\
-1.95]^{*}\end{array}$ & & $\begin{array}{l}-2.14[-3.80 \\
-0.48]^{*}\end{array}$ & & \\
\hline$p$ & $.043^{\mathrm{a}}$ & $<.001^{\mathrm{a}}$ & .059 & .016 & .672 & .551 \\
\hline $\mathrm{BIC}$ & 785.18 & 915.83 & 823.78 & 705.81 & 622.44 & 636.92 \\
\hline $\begin{array}{l}\text { Number of } \\
\text { observations }\end{array}$ & 110 & 108 & 109 & 77 & 78 & 77 \\
\hline \multirow[t]{2}{*}{$N$} & 51 & 51 & 51 & 50 & 51 & 51 \\
\hline & \multicolumn{6}{|c|}{ Pretherapy to posttherapy } \\
\hline Intercept & $\begin{array}{c}23.31[19.53 \\
27.08]^{*}\end{array}$ & $\begin{array}{c}58.91[50.79 \\
67.03]^{*}\end{array}$ & $\begin{array}{c}14.94[11.36 \\
18.52]^{*}\end{array}$ & $\begin{array}{c}52.99[43.47 \\
62.50]^{*}\end{array}$ & $\begin{array}{c}83.08[77.18 \\
88.98]^{*}\end{array}$ & $\begin{array}{c}53.21[46.66 \\
59.75]^{*}\end{array}$ \\
\hline Week & $\begin{array}{l}-0.17[-0.32 \\
-0.03]^{*}\end{array}$ & $\begin{array}{l}-1.05[-1.52 \\
-0.59]^{*}\end{array}$ & & & & \\
\hline$p$ & .021 & $<.001^{\mathrm{a}}$ & .135 & .074 & .179 & .474 \\
\hline $\mathrm{BIC}$ & 733.42 & 902.91 & 767.11 & 368.95 & 352.95 & 356.69 \\
\hline $\begin{array}{l}\text { Number of } \\
\text { observations }\end{array}$ & 115 & 116 & 111 & 42 & 42 & 42 \\
\hline \multirow[t]{2}{*}{$N^{\mathrm{b}}$} & 28 & 28 & 28 & 26 & 26 & 26 \\
\hline & \multicolumn{6}{|c|}{ Preprogram (baseline) to follow-up } \\
\hline Intercept & $\begin{array}{c}31.00[28.60 \\
33.40]^{*}\end{array}$ & $\begin{array}{c}75.28[70.80 \\
79.77]^{*}\end{array}$ & $\begin{array}{c}23.80[20.61 \\
26.98]^{*}\end{array}$ & $\begin{array}{c}71.30[64.66 \\
77.94]^{*}\end{array}$ & $\begin{array}{l}77.16[73.41 \\
\quad 80.90]^{*}\end{array}$ & $\begin{array}{l}51.11[47.23 \\
54.99]^{*}\end{array}$ \\
\hline Week & $\begin{array}{l}-0.85[-1.17 \\
-0.54]^{*}\end{array}$ & $\begin{array}{l}-2.47[-3.10 \\
-1.83]^{*}\end{array}$ & $\begin{array}{c}-0.60[-0.85 \\
-0.36]^{*}\end{array}$ & $\begin{array}{l}-0.88[-1.22 \\
-0.53]^{*}\end{array}$ & $\begin{array}{l}0.31[0.08 \\
0.54]^{*}\end{array}$ & \\
\hline$p$ & $<.001^{\mathrm{a}}$ & $<.001^{\mathrm{a}}$ & $<.001^{\mathrm{a}}$ & $<.001$ & .011 & .292 \\
\hline $\mathrm{BIC}$ & 1500.65 & 1843.84 & 1582.51 & 1051.63 & 948.43 & 966.8 \\
\hline $\begin{array}{l}\text { Number of } \\
\text { observations }\end{array}$ & 225 & 224 & 220 & 119 & 120 & 119 \\
\hline$N$ & 51 & 51 & 51 & 51 & 51 & 51 \\
\hline
\end{tabular}

${ }^{a}$ Final model included random slopes.

${ }^{\mathrm{b}}$ Only includes participants who completed the pretherapy assessment.

* 0 outside the confidence interval.

Note. 95\% confidence intervals are reported in brackets. $p$-values reported are for the change in model fit compared to a previously tested model. AAQ-II = Acceptance and Action Questionnaire-II; BAFT = Believability of Anxious Feelings and Thoughts Questionnaire; OQ45 = Outcome Questionnaire-45.2; DASS-21 = Depression Anxiety Stress Scales; QOLS = Quality of Life Scale; VLQ = Valued Living Questionnaire. 
Table 3

Means, Standard Deviations (SDs), and Effect Sizes From Preprogram (Baseline) to Follow-up

\begin{tabular}{|c|c|c|c|c|c|c|c|c|}
\hline & \multicolumn{5}{|c|}{ Means and SDs } & \multicolumn{3}{|c|}{ Hedges' $g$} \\
\hline & $\begin{array}{c}\text { Pre- } \\
\text { program } \\
\text { (baseline) }\end{array}$ & $\begin{array}{c}\text { Mid- } \\
\text { program }\end{array}$ & $\begin{array}{l}\text { Post- } \\
\text { program } \\
\text { /pre- } \\
\text { therapy }\end{array}$ & $\begin{array}{l}\text { Post- } \\
\text { therapy }\end{array}$ & Follow-up & $\begin{array}{l}\text { Preprogram to } \\
\text { postprogram }\end{array}$ & $\begin{array}{l}\text { Pretherapy to } \\
\text { posttherapy }\end{array}$ & $\begin{array}{l}\text { Preprogram } \\
\text { (baseline) to } \\
\text { follow-up }\end{array}$ \\
\hline AAQ-II & $\begin{array}{l}31.33 \\
(8.85)\end{array}$ & $\begin{array}{l}29.23 \\
(9.52)\end{array}$ & $\begin{array}{c}27.90 \\
(11.35)\end{array}$ & $\begin{array}{l}21.06 \\
(8.26)\end{array}$ & $\begin{array}{c}20.16 \\
(10.55)\end{array}$ & $-0.36[0.82,0.10]$ & $-0.65[-1.27,-0.03]$ & $-1.19[-1.71,-0.67]$ \\
\hline BAFT & $\begin{array}{c}77.65 \\
(16.30)\end{array}$ & $\begin{array}{c}71.10 \\
(19.05)\end{array}$ & $\begin{array}{c}65.07 \\
(23.03)\end{array}$ & $\begin{array}{c}43.71 \\
(17.68)\end{array}$ & $\begin{array}{c}43.48 \\
(17.71)\end{array}$ & $-0.67[-1.14,-0.19]$ & $-0.99[-1.63,-0.35]$ & $-2.04[-2.62,-1.45]$ \\
\hline DASS-21 & $\begin{array}{c}24.06 \\
(12.48)\end{array}$ & $\begin{array}{c}22.69 \\
(10.85)\end{array}$ & $\begin{array}{c}20.48 \\
(13.61)\end{array}$ & $\begin{array}{r}14.94 \\
(9.38)\end{array}$ & $\begin{array}{c}12.96 \\
(10.07)\end{array}$ & $-0.29[-0.75,0.17]$ & $-0.45[-1.06,0.17]$ & $-0.95[-1.46,-0.45]$ \\
\hline OQ-45 & $\begin{array}{c}73.98 \\
(23.68)\end{array}$ & - & $\begin{array}{c}65.25 \\
(26.02)\end{array}$ & $\begin{array}{c}54.76 \\
(20.59)\end{array}$ & $\begin{array}{c}53.52 \\
(26.75)\end{array}$ & $-0.36[-0.83,0.11]$ & $-0.43[-1.04,0.19]$ & $-0.83[-1.33,-0.33]$ \\
\hline QOLS & $\begin{array}{c}77.14 \\
(13.73)\end{array}$ & - & $\begin{array}{c}78.25 \\
(13.65)\end{array}$ & $\begin{array}{c}81.65 \\
(16.10)\end{array}$ & $\begin{array}{c}83.88 \\
(15.87)\end{array}$ & $0.09[-0.38,0.55]$ & $0.23[-0.38,0.84]$ & $0.47[-0.02,0.96]$ \\
\hline VLQ & $\begin{array}{c}50.42 \\
(14.66)\end{array}$ & - & $\begin{array}{c}50.72 \\
(16.90)\end{array}$ & $\begin{array}{c}55.25 \\
(15.94)\end{array}$ & $\begin{array}{c}53.42 \\
(18.90)\end{array}$ & $0.02[-0.44,0.49]$ & $0.27[-0.34,0.88]$ & $0.19[-0.30,0.67]$ \\
\hline
\end{tabular}

Note. The OQ-45, QOLS, and VLQ were not given at the midprogram assessment. 95\% confidence intervals are reported in brackets. Large effect sizes are bolded, and medium effect sizes are italicized. AAQ-II = Acceptance and Action Questionnaire- II; BAFT = Believability of Anxious Feelings and Thoughts Questionnaire; DASS-21 = Depression Anxiety Stress Scales; OQ-45 = Outcome Questionnaire-45.2; QOLS = Quality of Life Scale; VLQ = Valued Living Questionnaire. 
Table 4

$\underline{\text { Rates of Clinically Significant and Reliable Change }}$

\begin{tabular}{|c|c|c|c|c|c|c|c|c|c|c|c|c|c|c|c|c|c|}
\hline \multicolumn{6}{|c|}{ Postprogram } & \multicolumn{6}{|c|}{ Posttherapy } & \multicolumn{6}{|c|}{ One-month follow-up } \\
\hline \multicolumn{3}{|c|}{$\begin{array}{l}\text { Clinically significant } \\
\text { change }\end{array}$} & \multicolumn{3}{|c|}{ Reliable change $^{\mathrm{a}}$} & \multicolumn{3}{|c|}{$\begin{array}{c}\text { Clinically significant } \\
\text { change }\end{array}$} & \multicolumn{3}{|c|}{ Reliable change $^{\mathrm{b}}$} & \multicolumn{3}{|c|}{$\begin{array}{c}\text { Clinically significant } \\
\text { change }\end{array}$} & \multicolumn{3}{|c|}{ Reliable change $^{\mathrm{a}}$} \\
\hline \multicolumn{18}{|c|}{ AAQ-II } \\
\hline Yes & 11 & $37.9 \%$ & Yes & 9 & $31.0 \%$ & Yes & 11 & $64.7 \%$ & Yes & 13 & $81.3 \%$ & Yes & 19 & $76.0 \%$ & Yes & 15 & $60.0 \%$ \\
\hline No & 18 & $62.1 \%$ & No & 20 & $69.0 \%$ & No & 6 & $35.3 \%$ & No & 3 & $18.8 \%$ & No & 6 & $24.0 \%$ & No & 10 & $40.0 \%$ \\
\hline & & & & & & & & BAFT & & & & & & & & & \\
\hline Yes & 23 & $79.3 \%$ & Yes & 17 & $63.0 \%$ & Yes & 17 & $100 \%$ & Yes & 12 & $75.0 \%$ & Yes & 25 & $100 \%$ & Yes & 22 & $91.7 \%$ \\
\hline No & 6 & $20.7 \%$ & No & 10 & $37.0 \%$ & No & 0 & $0 \%$ & No & 4 & $25.0 \%$ & No & 0 & $0 \%$ & No & 2 & $8.3 \%$ \\
\hline \multicolumn{18}{|c|}{ DASS-21 } \\
\hline Yes & 22 & $75.9 \%$ & Yes & 6 & $20.7 \%$ & Yes & 17 & $100 \%$ & Yes & 9 & $56.3 \%$ & Yes & 22 & $88.0 \%$ & Yes & 18 & $72.0 \%$ \\
\hline No & 7 & $24.1 \%$ & No & 23 & $79.3 \%$ & No & 0 & $0 \%$ & No & 7 & $43.8 \%$ & No & 3 & $12.0 \%$ & No & 7 & $28.0 \%$ \\
\hline \multicolumn{18}{|c|}{ OQ-45 } \\
\hline Yes & 22 & $78.6 \%$ & Yes & 27 & $100 \%$ & Yes & 16 & $94.1 \%$ & Yes & 15 & $100 \%$ & Yes & 22 & $88.0 \%$ & Yes & 19 & $79.2 \%$ \\
\hline No & 6 & $21.4 \%$ & No & 0 & $0 \%$ & No & 1 & $5.9 \%$ & No & 0 & $0 \%$ & No & 3 & $12.0 \%$ & No & 5 & $20.8 \%$ \\
\hline
\end{tabular}

${ }^{\text {a }}$ Compared to preprogram.

${ }^{\mathrm{b}}$ Compared to pretherapy.

Note. AAQ-II = Acceptance and Action Questionnaire-II; BAFT = Believability of Anxious Feelings and Thoughts Questionnaire; OQ-45 = Outcome Questionnaire-45.2; DASS-21 = Depression Anxiety Stress Scales; QOLS = Quality of Life Scale; VLQ = Valued Living Questionnaire. 


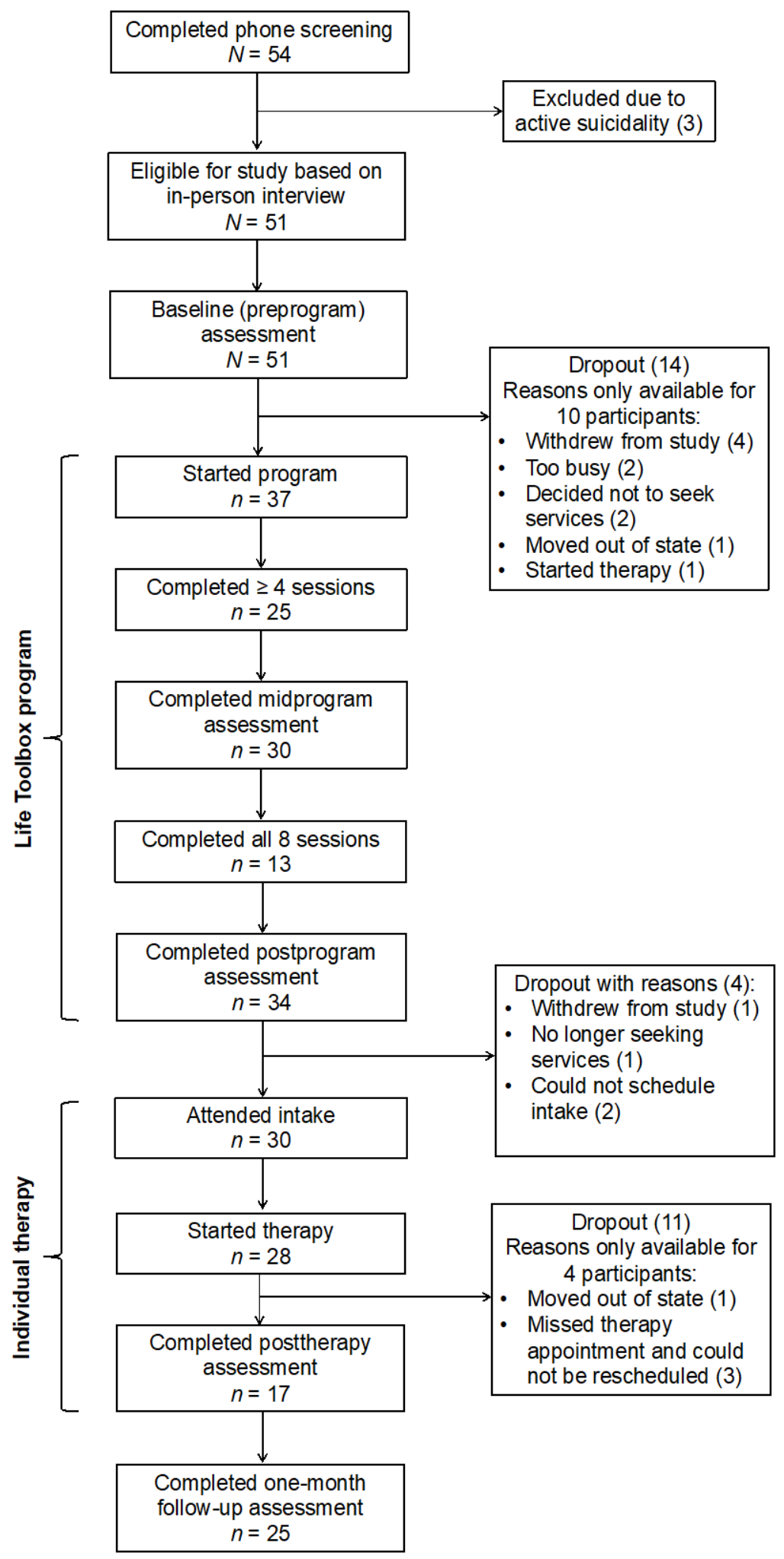

Figure 1. Participant flow and attrition. Reasons for dropout are only provided for participants who directly contacted researchers; the reasons are unknown for other participants. 